\title{
Concave measures and the fuzzy core of exchange economies with heterogeneous divisible commodities
}

\author{
Farhad Hüsseinov ${ }^{\mathrm{b}}$, Nobusumi Sagara ${ }^{\mathrm{b}, *}$ \\ ${ }^{a}$ Department of Economics, Bilkent University, 06800 Bilkent, Ankara, Turkey \\ ${ }^{\mathrm{b}}$ Faculty of Economics, Hosei University, 4342 Aihara, Machida, Tokyo 194-0298, Japan
}

Received 11 October 2010; received in revised form 29 October 2011; accepted 30 December 2011

Available online 10 January 2012

\begin{abstract}
The main purpose of this paper is to prove the existence of the fuzzy core of an exchange economy with a heterogeneous divisible commodity in which preferences of individuals are given by nonadditive utility functions defined on a $\sigma$-algebra of admissible pieces of the total endowment of the commodity. The problem is formulated as the partitioning of a measurable space among finitely many individuals. Applying the Yosida-Hewitt decomposition theorem, we also demonstrate that partitions in the fuzzy core are supportable by prices in $L^{1}$.
\end{abstract}

(C) 2012 Elsevier B.V. All rights reserved.

Keywords: Nonatomic vector measure; Concave measure; Fuzzy coalition; Fuzzy core; Supporting price; Yosida-Hewitt decomposition

\section{Introduction}

Cooperative fuzzy games proposed by Aubin [2,3] allow for partial participation of individuals in coalitions. In defining the fuzzy core of exchange economies with homogeneous divisible commodities, individuals contribute only some portions of their initial endowments to coalitions they belong to. That is, a fuzzy coalition unlike the classical (crisp) coalitions, does not necessarily require its participants to contribute the whole of their initial endowments. A remarkable result for exchange economies established by Aubin states that under the standard assumptions of continuous, convex preferences the fuzzy core and the set of Walrasian allocations coincide (see also [12,14]).

In this paper we study the fuzzy core of an exchange economy with a heterogeneous divisible commodity in which preferences of individuals are given by set functions defined in a $\sigma$-algebra of admissible pieces of the total endowment of the commodity. Following the traditions of fair division literature along the lines of Dubins and Spanier [9], a heterogeneous divisible commodity is modeled as a nonatomic finite measure space. The total endowment of the heterogeneous commodity is metaphorically called a "cake" in this literature and the problem of fair division consists in partitioning the cake among a finite number of individuals according some criteria of fairness and efficiency.

\footnotetext{
* Corresponding author. Tel.: +81 42783 2556; fax: +81 427832611.

E-mail addresses: farhad@bilkent.edu.tr (F. Hüsseinov), nsagara@ hosei.ac.jp (N. Sagara).
} 
A common assumption in the theory of fair division is that the preferences of each individual are represented by a nonatomic probability measure. Under this additive utility hypothesis, Lyapunov's convexity theorem (see [19]) guarantees the convexity and compactness of the utility possibility set, crucial to establishing the existence and characterization of various solutions. However, here we assume preferences to be represented by nonadditive utility functions; hence the utility possibility set does not necessarily possess these properties. The utility functions assumed here contain concave measures introduced by Sagara and Vlach [21-23].

Hüsseinov [15,17] and Sagara and Vlach [24] proved the existence of the core with nonadditive evaluations for exchange economies with heterogeneous divisible commodity under diverse assumptions. The corresponding result for the case of the fuzzy core is not straightforward; an adequate notion of fuzzy improvement must reflect awareness of agents of the bounds of the available heterogeneous divisible commodity. We propose here a such notion of the fuzzy improvement and the fuzzy core.

The organization of the paper is as follows: In Section 2 we present a representation result for concave measures, stating that an arbitrary concave measure can be represented as a composition of a concave function and a finitedimensional nonatomic vector measure. From this characterization we derive the continuity of concave measures at the measurable set whose vector measure lies in the interior of Lyapunov's set. We also provide a core representation theorem for nonatomic vector measure games along the lines of Einy et al. [11].

Section 3 is devoted to the formulation of the fuzzy coalitions and fuzzy core. To this end, we focus our attention on the case where the single heterogeneous divisible commodity possesses a finite number of attributes, which can be evaluated objectively in terms of finite-dimensional nonatomic vector measures. We define the notions of the fuzzy coalitions and fuzzy core in an exchange economy with a heterogeneous divisible commodity, where each individual has a utility function represented by a nonadditive set function.

The main result of this paper, Theorem 4.2 on the existence of the fuzzy core, is stated in Section 4. To prove this theorem, we extend the commodity space from the set of measurable sets to the set of measurable functions taking values in the unit interval along the lines of Akin [1], Dall'Aglio [7] and Dvoretsky et al. [10]. If $f$ in $L^{\infty}$ is a characteristic function of a measurable set $A$, then an individual possessing $f$ is fully entitled to set $A$ and to nothing else. Thus, we can treat allocations in the extended economy with an $L^{\infty}$-commodity space, which can be embedded into the framework of Bewley [5,6]. We prove the existence of the fuzzy core of this extended economy by constructing a nontransferrable utility (NTU) game and showing that it satisfies the assumptions of Scarf's core existence theorem (see [25]). Exploiting a technique from Lindenstrauss [18], the existence of the fuzzy core in the original exchange economy follows from the observation that the extreme points in the fuzzy core of the extended economy are indeed measurable partitions.

Section 5 deals with the supportability of efficient partitions by prices. The argument is based on the effective use of the separation theorem under the convexity assumption. We demonstrate that partitions in the fuzzy core are supportable by prices in $L^{1}$, applying the Yosida-Hewitt decomposition theorem (see [26]), which is by now a standard method, having been established by Bewley [5,6].

Appendix provides a supplementary result on the extension of continuous, quasiconcave, strictly monotonic functions on a compact convex subset of the nonnegative orthant to the entire domain.

\section{Representation and continuity of concave measures}

\subsection{Representation of concave measures}

Let $(\Omega, \mathcal{F})$ be a measurable space with a $\sigma$-algebra $\mathcal{F}$ of subsets of a nonempty set $\Omega$. A measure $\mu$ on $\mathcal{F}$ is nonatomic if, for every $A \in \mathcal{F}$ with $\mu(A)>0$, there exists $E \in \mathcal{F}$ with $E \subset A$ such that $0<\mu(E)<\mu(A)$. For nonatomic finite measures $\mu_{1}, \ldots, \mu_{m}$, we denote by $\vec{\mu}=\left(\mu_{1}, \ldots, \mu_{m}\right)$ an $\mathbb{R}^{m}$-valued vector measure. Lyapunov's convexity theorem asserts that the range $\mathcal{R}(\vec{\mu})$ of $\vec{\mu}$ is a compact and convex set in $\mathbb{R}^{m}$ (see [19]).

For an arbitrarily given $A \in \mathcal{F}$ and $t \in[0,1]$, we define the family $\mathcal{K}_{t}^{\vec{\mu}}(A)$ of measurable subsets of $A$ by:

$$
\mathcal{K}_{t}^{\vec{\mu}}(A)=\{E \in \mathcal{F} \mid E \subset A \text { and } \vec{\mu}(E)=t \vec{\mu}(A)\} .
$$

By Lyapunov's convexity theorem, $\mathcal{K}_{t}^{\vec{\mu}}(A)$ is nonempty for every $A \in \mathcal{F}$ and $t \in[0,1]$. Furthermore, for an arbitrarily given $A, B \in \mathcal{F}$ and $t \in[0,1]$, we denote by $\mathcal{K}_{t}^{\vec{\mu}}(A, B)$ the family of sets $C \in \mathcal{F}$, which are written as the union of some 
disjoint sets $E \in \mathcal{K}_{t}^{\vec{\mu}}(A)$ and $F \in \mathcal{K}_{1-t}^{\vec{\mu}}(B)$. For a nonatomic scalar measure $\mu$ we use notation $\mathcal{K}_{t}^{\mu}(A, B)$. It is evident that $C \in \mathcal{K}_{t}^{\vec{\mu}}(A, B)$ if and only if $C \in \mathcal{K}_{t}^{\mu_{k}}(A, B)$ for each $k=1, \ldots, m$, and hence, $\mathcal{K}_{t}^{\vec{\mu}}(A, B)=\bigcap_{k=1}^{m} \mathcal{K}_{t}^{\mu_{k}}(A, B)$ for every $A, B \in \mathcal{F}$ and $t \in[0,1]$. It can be shown that $\mathcal{K}_{t}^{\vec{\mu}}(A, B)$ is nonempty for every $A, B \in \mathcal{F}$ and $t \in[0,1]$ (see [23]).

The notion of concave measures on $\sigma$-algebras presented in the following definition bears an obvious resemblance to that of concave functions on real vector spaces. We extend here the definition of Sagara and Vlach [21-23] to the vector measure case.

Definition 2.1. A set function $v: \mathcal{F} \rightarrow \mathbb{R}$ is a concave measure if $v(\emptyset)=0$ and there exists a finite-dimensional nonatomic vector measure $\vec{\mu}$ such that for every $A, B \in \mathcal{F}$ and $t \in[0,1]$, we have

$$
t v(A)+(1-t) v(B) \leq v(C) \text { for every } C \in \mathcal{K}_{t}^{\vec{\mu}}(A, B) .
$$

When the underlying vector measure is $\vec{\mu}$ for the concave measure $v$, we say that $v$ is a $\vec{\mu}$-concave measure.

The following result presents a useful representation of concave measures.

Theorem 2.1. A set function $v: \mathcal{F} \rightarrow \mathbb{R}$ is a concave measure if and only if there exist a finite-dimensional nonatomic vector measure $\vec{\mu}$ and a concave function $\varphi: \mathcal{R}(\vec{\mu}) \rightarrow \mathbb{R}$ with $\varphi(\mathbf{0})=0$ such that $v=\varphi \circ \vec{\mu}$.

Proof. Suppose that $v$ is a $\vec{\mu}$-concave measure. For the construction of a concave function asserted in the theorem, it suffices to show that if $\vec{\mu}(A)=\vec{\mu}(B)$, then $v(A)=v(B)$, because if this is the case, the value $v(A)$ depends exclusively on $\vec{\mu}(A)$, which implies that $v(A)=\varphi(\vec{\mu}(A))$ for the real-valued function $\varphi$ defined on the compact convex set $\mathcal{R}(\vec{\mu})$ as $\varphi(x)=v(A)$, where $A \in \mathcal{F}$ and $\vec{\mu}(A)=x$. The concavity of $\varphi$ follows from that of $v$.

Suppose that $\vec{\mu}(A)=\vec{\mu}(B)$. Let $A_{1} \subset A \backslash B$ and $B_{1} \subset B \backslash A$ be such that $\vec{\mu}\left(A_{1}\right)=\frac{1}{2} \vec{\mu}(A \backslash B)$ and $\vec{\mu}\left(B_{1}\right)=\frac{1}{2} \vec{\mu}(B \backslash A)$, which is possible by Lyapunov's convexity theorem. Define $C=A_{1} \cup B_{1} \cup(A \cap B)$ and $D=(A \cup B) \backslash\left(A_{1} \cup B_{1}\right)$. Then, $\vec{\mu}(C)=\vec{\mu}(D)$ and $C, D \in \mathcal{K}_{1 / 2}^{\vec{\mu}}(A, B)$. It is easily seen that also $A, B \in \mathcal{K}_{1 / 2}^{\vec{\mu}}(C, D)$. By the concavity of $v$, we have

$$
\begin{aligned}
& v(A) \geq \frac{1}{2}(v(C)+v(D)), \quad v(B) \geq \frac{1}{2}(v(C)+v(D)), \\
& v(C) \geq \frac{1}{2}(v(A)+v(B)) \quad \text { and } \quad v(D) \geq \frac{1}{2}(v(A)+v(B)) .
\end{aligned}
$$

Summing these inequalities up we will have an inequality where the left and right hand sides are the same. Therefore, all the inequalities in the above are in fact equalities. It follows from this observation that $v(A)=v(B)$.

The converse implication that $v=\varphi \circ \vec{\mu}$ is a concave measure immediately follows from the concavity of $\varphi$ and $\varphi(\mathbf{0})=0$.

Recall that a set function $v: \mathcal{F} \rightarrow \mathbb{R}$ is submodular if $v(A \cup B)+v(A \cap B) \leq v(A)+v(B)$ for every $A, B \in \mathcal{F}$. The next example is due to Sagara and Vlach [24].

Example 2.1. Let $\mu$ be a nonatomic scalar measure. Define the set function $v_{\varphi}: \mathcal{F} \rightarrow \mathbb{R}$ for a continuous function $\varphi: \mathcal{R}(\mu) \rightarrow \mathbb{R}$ with $\varphi(0)=0$ by $v_{\varphi}=\varphi \circ \mu$. The following conditions are equivalent:

(i) $\varphi$ is concave;

(ii) $v_{\varphi}$ is $\mu$-concave;

(iii) $v_{\varphi}$ is submodular.

The equivalence in the example is not true for the vector measure case. Indeed, for $m=2$, one can construct an example in which there exists a continuous concave function $\varphi$ with $\varphi(\mathbf{0})=0$ such that $v_{\varphi}$ is not $\vec{\mu}$-concave. For details, see Sagara and Vlach [21]. 


\subsection{Continuity of concave measures}

Sets $A$ and $B$ in $\mathcal{F}$ are $\vec{\mu}$-equivalent if $\vec{\mu}(A \triangle B)=0$, where $A \triangle B=(A \cup B) \backslash(A \cap B)$ is the symmetric difference of $A$ and $B$. The $\vec{\mu}$-equivalence defines an equivalence relation (reflexive, symmetric, transitive binary relation) on $\mathcal{F}$. We denote the $\vec{\mu}$-equivalence class of $A \in \mathcal{F}$ by $[A]$ and denote the set of $\vec{\mu}$-equivalence classes in $\mathcal{F}$ by $\mathcal{F}_{\vec{\mu}}$. Define the metric $d_{\vec{\mu}}$ on $\mathcal{F}_{\vec{\mu}}$ by $d_{\vec{\mu}}([A],[B])=\|\vec{\mu}(A \triangle B)\|$, where $\|\cdot\|$ is the Euclidean norm of $\mathbb{R}^{m}$. If $\mathcal{F}$ is countably generated, then the metric space $\left(\mathcal{F}_{\vec{\mu}}, d_{\vec{\mu}}\right)$ is complete and separable (see [8, Lemma III.7.113, Theorem 40.B13, Theorem 40.B]).

Continuous functions on $\left(\mathcal{F}_{\vec{\mu}}, d_{\vec{\mu}}\right)$ arise in a natural way from the set functions on $\mathcal{F}$. The following definition is a straightforward generalization of Sagara and Vlach $[21,22]$ to the vector measure case.

Definition 2.2. A set function $v: \mathcal{F} \rightarrow \mathbb{R}$ is $\vec{\mu}$-continuous at $A \in \mathcal{F}$ if for every $\varepsilon>0$ there exists $\delta>0$ such that $\|\vec{\mu}(A \triangle B)\|<\delta$ implies $|v(A)-v(B)|<\varepsilon$. When $v$ is $\vec{\mu}$-continuous at every element of $\mathcal{F}$ we say that $v$ is $\vec{\mu}$-continuous.

The $\vec{\mu}$-continuity of set functions on $\mathcal{F}$ is stronger than the continuity of those in the measure-theoretic sense: $v$ is continuous at $A \in \mathcal{F}$ if for every sequence $\left\{A^{q}\right\}$ in $\mathcal{F}$ with $A^{q} \uparrow A$ or $A^{q} \downarrow A$, we have $\lim _{q} v\left(A^{q}\right)=v(A)$.

We denote by int $\mathcal{R}(\vec{\mu})$ and bd $\mathcal{R}(\vec{\mu})$ the interior of $\mathcal{R}(\vec{\mu})$ and the boundary of $\mathcal{R}(\vec{\mu})$, respectively.

Corollary 2.1. Every $\vec{\mu}$-concave measure is $\vec{\mu}$-continuous at every $A \in \mathcal{F}$ with $\vec{\mu}(A) \in \operatorname{int} \mathcal{R}(\vec{\mu})$.

Proof. By Theorem 2.1, any concave measure $v$ is of the form $v=\varphi \circ \vec{\mu}$, where $\varphi: \mathcal{R}(\vec{\mu}) \rightarrow \mathbb{R}$ is a concave function. Let $\left\{A^{q}\right\}$ be a sequence in $\mathcal{F}$ with $\vec{\mu}\left(A^{q} \triangle A\right) \rightarrow 0$. Then we have $\vec{\mu}\left(A^{q}\right) \rightarrow \vec{\mu}(A)$ because of the inequality $-\mu_{k}\left(A^{q} \triangle A\right) \leq \mu_{k}\left(A^{q}\right)-\mu_{k}(A) \leq \mu_{k}\left(A^{q} \triangle A\right)$ for each $k=1, \ldots, m$. Thus, $v\left(A^{q}\right) \rightarrow v(A)$ since $\varphi$ is continuous on the interior of its domain as a concave function.

We denote by $b a(\Omega, \mathcal{F})$ the space of bounded, finitely additive, signed measures on $\mathcal{F}$. A set function $v: \mathcal{F} \rightarrow \mathbb{R}$ is a game if $v(\emptyset)=0$. A feasible payoff of a game $v$ is an element $\mu$ in $b a(\Omega, \mathcal{F})$ satisfying $\mu(\Omega)=v(\Omega)$. The core of a game $v$ is defined by

$$
\mathcal{C}(v)=\{\mu \in b a(\Omega, \mathcal{F}) \mid v \leq \mu \text { and } \mu(\Omega)=v(\Omega)\},
$$

that is, the core is the set of feasible payoffs upon which no coalition can improve.

Recall that a supergradient of a concave function $\varphi: \mathcal{R}(\vec{\mu}) \rightarrow \mathbb{R}$ at $x \in \mathcal{R}(\vec{\mu})$ is a vector $p \in \mathbb{R}^{m}$ satisfying $\varphi(y)-\varphi(x) \leq\langle p, y-x\rangle$ for every $y \in \mathcal{R}(\vec{\mu})$, where $\langle\cdot, \cdot\rangle$ is the inner product in $\mathbb{R}^{m}$. The superdifferential $\partial \varphi(x)$ of $\varphi$ at $x$ is the set of supergradients of $\varphi$ at $x$.

Theorem 2.2. If $v: \mathcal{F} \rightarrow \mathbb{R}$ is a $\vec{\mu}$-concave measure that is $\vec{\mu}$-continuous at $\Omega$, then there exists a concave function $\varphi: \mathcal{R}(\vec{\mu}) \rightarrow \mathbb{R}$ with $\varphi(\mathbf{0})=0$ such that:

$$
\mathcal{C}(v)=\{\langle p, \vec{\mu}\rangle \in b a(\Omega, \mathcal{F}) \mid p \in \partial \varphi(\vec{\mu}(\Omega)),\langle p, \vec{\mu}(\Omega)\rangle=\varphi(\vec{\mu}(\Omega))\} .
$$

For proving the theorem, we will need the following result by Einy et al. [11].

Lemma 2.1. Let $\vec{\mu}$ be a finite-dimensional nonatomic vector measure and $\varphi: \mathcal{R}(\vec{\mu}) \rightarrow \mathbb{R}$ be a concave function with $\varphi(\mathbf{0})=0$ that is continuous at $\vec{\mu}(\Omega)$. Then the core of the game $v=\varphi \circ \vec{\mu}$ is given by (2.1).

Proof of Theorem 2.2. Let $v$ be a $\vec{\mu}$-concave measure that is $\vec{\mu}$-continuous at $\Omega$. Then $v=\varphi \circ \vec{\mu}$ for some concave function $\varphi$ on $\mathcal{R}(\vec{\mu})$ by Theorem 2.1. Since the $\vec{\mu}$-continuity of $v=\varphi \circ \vec{\mu}$ at $\Omega$ implies the continuity at $\Omega$ in the sense that $v\left(A^{q}\right) \rightarrow v(\Omega)$ for every sequence $\left\{A^{q}\right\}$ in $\mathcal{F}$ with $A^{q} \uparrow \Omega$, the result is an immediate consequence of Lemma 2.1 .

Theorem 2.2 involves a "core representation" result for $\vec{\mu}$-concave measures. Indeed, the core of a $\vec{\mu}$-concave measure $v=\varphi \circ \vec{\mu}$ that is $\vec{\mu}$-continuous at $\Omega$ can be characterized by the local behavior of the superdifferential of $\varphi$ at $\vec{\mu}(\Omega)$. This conforms with a similar characterization of the core of a game with the form $v=\varphi \circ \vec{\mu}$ obtained by Einy et al. [11] under the alternative continuity hypothesis. 


\section{Fuzzy coalitions in exchange economies}

\subsection{Partitioning of a measurable space}

The problem of dividing a heterogeneous commodity among a finite number of individuals is formulated as partitioning a measurable space $(\Omega, \mathcal{F})$. Here, set $\Omega$ is a heterogeneous divisible commodity, and $\sigma$-algebra $\mathcal{F}$ of subsets of $\Omega$ describes the collection of possible pieces of $\Omega$. There are $m$ attributes for the heterogeneous divisible commodity $\Omega$, each of which has a cardinal evaluation represented by a nonatomic finite measure $\mu_{k}$ on $(\Omega, \mathcal{F})$ for $k=1, \ldots, m$. We assume in the sequel that $\vec{\mu}=\left(\mu_{1}, \ldots, \mu_{m}\right)$ is a nonatomic vector measure such that its component measures are mutually absolutely continuous.

There are $n$ individuals, indexed by $i=1, \ldots, n$, with the set $N=\{1, \ldots, n\}$ of all individuals, whose preferences on $\mathcal{F}$ are given by utility functions $v_{i}: \mathcal{F} \rightarrow \mathbb{R}$ for $i \in N$. A partition of $\Omega$ is an ordered $n$-tuple $\left(A_{1}, \ldots, A_{n}\right)$ of mutually disjoint elements $A_{1}, \ldots, A_{n}$ in $\mathcal{F}$ whose union is $\Omega$, where each $A_{i}$ is a piece of the cake assigned to individual $i$. Let individual $i$ be initially endowed with $\Omega_{i} \in \mathcal{F}$ for $i \in N$. So $\left(\Omega_{1}, \ldots, \Omega_{n}\right)$ is an initial partition of $\Omega$. An exchange economy $\mathcal{E}=\left\langle(\Omega, \mathcal{F}), \Omega_{i}, v_{i}\right\rangle_{i \in N}$ for the partitioning problem under study is the primitive consisting of a common consumption set $(\Omega, \mathcal{F})$ and the individuals' profile of initial endowments $\Omega_{i}$ and utility functions $v_{i}$.

We formulate partial participation of individuals to coalitions as proposed by Aubin [2,3]. A nonzero vector $\alpha=$ $\left(\alpha_{1}, \ldots, \alpha_{n}\right)$ in the unit cube $[0,1]^{n}$ is called a fuzzy coalition, whose component $\alpha_{i} \in[0,1]$ denotes the degree of participation of individual $i$ in this coalition. For each nonempty set $S \subset N$, a fuzzy coalition with support $S$ is a vector $\alpha^{S}=\left(\alpha_{1}^{S}, \ldots, \alpha_{n}^{S}\right) \in[0,1]^{n}$, satisfying $\alpha_{i}^{S}>0$ for each $i \in S$ and $\alpha_{i}^{S}=0$ otherwise; $S$ is the set of 'active individuals' in the fuzzy coalition $\alpha^{S}$. The vector $e^{S}$ in $\{0,1\}^{n}$ defined as $e_{i}^{S}=1$ for each $i \in S$ and $e_{i}^{S}=0$ otherwise is called a crisp coalition, and is identified with an ordinary (nonfuzzy) coalition $S$.

Definition 3.1. A partition $\left(A_{1}, \ldots, A_{n}\right)$ is an $\alpha^{S}$-partition if for each $i \in S$ there exist $E_{i} \in \mathcal{K}_{\alpha_{i}^{S}}^{\vec{\mu}}\left(A_{i}\right)$ and $F_{i} \in \mathcal{K}_{\alpha_{i}^{S}}^{\vec{\mu}}\left(\Omega_{i}\right)$ such that

$$
\vec{\mu}\left(\bigcup_{i \in S} E_{i} \triangle \bigcup_{i \in S} F_{i}\right)=0 .
$$

An $e^{S}$-partition is simply said to be an $S$-partition.

It follows from the definition that an $S$-partition $\left(A_{1}, \ldots, A_{n}\right)$ satisfies the coalitional feasibility constraint character wise, that is

$$
\mu_{k}\left(\bigcup_{i \in S} A_{i} \triangle \bigcup_{i \in S} \Omega_{i}\right)=0
$$

for each $k=1, \ldots, m$.

Definition 3.2. A fuzzy coalition $\alpha^{S}$ improves upon a partition $\left(B_{1}, \ldots, B_{n}\right)$ if there exists an $\alpha^{S}$-partition $\left(A_{1}, \ldots, A_{n}\right)$ such that $v_{i}\left(A_{i}\right)>v_{i}\left(B_{i}\right)$ for each $i \in S$. A partition that cannot be improved upon by any fuzzy coalition is a fuzzy core partition.

\subsection{Allocations in $L^{\infty}$-spaces}

Define $\mu=\sum_{k=1}^{m} \mu_{k}$. Let $L^{\infty}(\Omega, \mathcal{F}, \mu)$ be the space of $\mu$-essentially bounded measurable functions on $\Omega$ with the sup norm. Denote by $\chi_{A} \in L^{\infty}(\Omega, \mathcal{F}, \mu)$ the characteristic function of $A \in \mathcal{F}$.

Let $\mathcal{X}=\left\{f \in L^{\infty}(\Omega, \mathcal{F}, \mu) \mid 0 \leq f \leq 1, \mu\right.$-a.e. $\}$. Then, $\mathcal{X}$ is a weakly* compact, convex subset of $L^{\infty}(\Omega, \mathcal{F}, \mu)$. We identify $\mathcal{F}$ with the subset of characteristic functions in $\mathcal{X}$. An $n$-tuple $\left(f_{1}, \ldots, f_{n}\right)$ of elements in $L^{\infty}(\Omega, \mathcal{F}, \mu)$ is an allocation of $\Omega$ if $\sum_{i=1}^{n} f_{i}=1$ and $f_{i} \in \mathcal{X}$ for each $i \in N$. Note that $\left(A_{1}, \ldots, A_{n}\right)$ is a partition of $\Omega$ if and only if $\sum_{i=1}^{n} \chi_{A_{i}}=1$. We denote by $\mathcal{A}$ the set of allocations of $\Omega$. 
For $f \in \mathcal{X}$, set $\mu_{k}(f)=\int f d \mu_{k}$ for $k=1, \ldots, m$. Given a utility function $v_{i}$ of the form $v_{i}=\varphi_{i} \circ \vec{\mu}$ with $\varphi_{i}: \mathcal{R}(\vec{\mu}) \rightarrow \mathbb{R}$, we will denote by $\hat{v}_{i}$ the extension of $v_{i}$ to $\mathcal{X}$ defined as $\hat{v}_{i}(f)=\varphi_{i}(\vec{\mu}(f))$. This extension is indeed well defined because $\mathcal{R}(\vec{\mu})$ coincides with the set $\{\vec{\mu}(f) \mid f \in \mathcal{X}\} \subset \mathbb{R}^{m}$ by Lyapunov's convexity theorem.

An exchange economy $\widehat{\mathcal{E}}=\left\langle\mathcal{X}, \chi_{\Omega_{i}}, \hat{v}_{i}\right\rangle_{i \in N}$ for the allocation problem is the primitive consisting of a common consumption set $\mathcal{X}$ and the individuals' profile of initial endowments $\chi_{\Omega_{i}}$ and utility functions $\hat{v}_{i}$, which is an extension of the original economy $\mathcal{E}=\left\langle(\Omega, \mathcal{F}), \Omega_{i}, v_{i}\right\rangle_{i \in N}$ where $v_{i}=\varphi_{i} \circ \vec{\mu}$ for each $i \in N$.

Lemma 3.1. If $\varphi: \mathcal{R}(\vec{\mu}) \rightarrow \mathbb{R}$ is continuous and (strictly) (quasi) concave on $\mathcal{R}(\vec{\mu})$, then function $\hat{v}: \mathcal{X} \rightarrow \mathbb{R}$ of the form $\hat{v}=\varphi \circ \vec{\mu}$ is weakly* continuous and (strictly) (quasi) concave.

Proof. Let $\left\{f^{q}\right\}$ be a net in $\mathcal{X}$ that converges weakly* to $f \in \mathcal{X}$. Since each measure $\mu_{k}$ is absolutely continuous with respect to $\mu$, there exists $g_{k} \in L^{1}(\Omega, \mathcal{F}, \mu)$ such that $\mu_{k}(A)=\int_{A} g_{k} d \mu$ for every $A \in \mathcal{F}$. Then we have $\lim _{q} \mu_{k}\left(f^{q}\right)=$ $\lim _{q} \int f^{q} g_{k} d \mu=\int f g_{k} d \mu=\mu_{k}(f)$ for each $k=1, \ldots, m$ because $f^{q} \rightarrow f$ in the weak* topology of $L^{\infty}(\Omega, \mathcal{F}, \mu)$. Therefore, $\lim _{q} \vec{\mu}\left(f^{q}\right)=\vec{\mu}(f)$. By the continuity of $\varphi$, we obtain $\lim _{q} \hat{v}\left(f^{q}\right)=\lim _{q} \varphi\left(\vec{\mu}\left(f^{q}\right)\right)=\varphi(\vec{\mu}(f))=\hat{v}(f)$. The (strict) (quasi)concavity of $\hat{v}$ follows immediately from that of $\varphi$ and the affinity of the mapping $\mathcal{X} \ni f \mapsto \vec{\mu}(f) \in \mathcal{R}(\vec{\mu})$.

Lemma 3.2. $\mathcal{A}$ is a weakly* compact, convex subset of $\left[L^{\infty}(\Omega, \mathcal{F}, \mu)\right]^{n}$.

To prove this lemma, we borrow from functional analysis the following characterization of weakly* compact sets. (For a proof, see [8, Corollary V.4.3].)

Proposition 3.1. Let $X$ be a Banach space and $X^{*}$ be its dual space. A subset of $X^{*}$ is weakly* compact if and only if it is closed in the weak* topology and bounded in the norm topology.

Proof of Lemma 3.2. The convexity and the norm boundedness of $\mathcal{A}$ are obvious. We shall show that $\mathcal{A}$ is weakly* closed in $\left[L^{\infty}(\Omega, \mathcal{F}, \mu)\right]^{n}$. Let $\left(f_{1}^{q}, \ldots, f_{n}^{q}\right)$ be a net in $\mathcal{A}$ that weakly* converges to $\left(f_{1}, \ldots, f_{n}\right)$. Suppose that $\sum_{i=1}^{n} f_{i} \neq 1$. Then there exists a set $A \in \mathcal{F}$ with $\mu(A)>0$ such that $\sum_{i=1}^{n} f_{i}(\omega)<1$ for $\omega \in A$ or $\sum_{i=1}^{n} f_{i}(\omega)>1$ for $\omega \in A$. Integrating these inequalities over $A$, we have $\sum_{i=1}^{n} \int f_{i} \chi_{A} d \mu \neq \mu(A)$, but the weak* convergence $f_{i}^{q} \rightarrow f_{i}$ in $L^{\infty}(\Omega, \mathcal{F}, \mu)$ implies that $\mu(A)=\int_{A}\left(\sum_{i=1}^{n} f_{i}^{q}\right) d \mu=\sum_{i=1}^{n} \lim _{q} \int f_{i}^{q} \chi_{A} d \mu=\sum_{i=1}^{n} \int f_{i} \chi_{A} d \mu \neq \mu(A)$, a contradiction. Therefore, $\sum_{i=1}^{n} f_{i}=1$. The proof of the nonnegativity of $f_{i}$ is similar. Hence, $\sum_{i=1}^{n} f_{i}=1$ and $f_{i} \in \mathcal{X}$ for each $i \in N$, which yields $\left(f_{1}, \ldots, f_{n}\right) \in \mathcal{A}$. The weak* compactness of $\mathcal{A}$ follows from Proposition 3.1.

To explore the notion of fuzzy core allocations for $\widehat{\mathcal{E}}=\left\langle\mathcal{X}, \chi_{\Omega_{i}}, \hat{v}_{i}\right\rangle_{i \in N}$, we introduce a set-valued mapping $\widehat{\mathcal{K}}_{t}^{\vec{\mu}}$ : $\mathcal{X} \rightarrow 2^{\mathcal{X}}$, an eligible extension of $\mathcal{K}_{t}^{\vec{\mu}}: \mathcal{F} \rightarrow 2^{\mathcal{F}}$, as follows. For $f \in \mathcal{X}$ and $t \in[0,1]$, we define:

$$
\widehat{\mathcal{K}}_{t}^{\vec{\mu}}(f)=\{v \in \mathcal{X} \mid \vec{\mu}(v)=t \vec{\mu}(f), v \leq f\} .
$$

It follows from the definition that $\mathcal{K}_{t}^{\vec{\mu}}(A) \subset \widehat{\mathcal{K}}_{t}^{\vec{\mu}}\left(\chi_{A}\right)$ for every $A \in \mathcal{F}$ and $t \in[0,1]$. Moreover, $\chi_{E} \in \widehat{\mathcal{K}}_{t}^{\vec{\mu}}\left(\chi_{A}\right)$ if and only if $E \in \mathcal{K}_{t}^{\vec{\mu}}(A)$.

Definition 3.3. An allocation $\left(f_{1}, \ldots, f_{n}\right)$ is an $\alpha^{S}$-allocation if for each $i \in S$ there exist $v_{i} \in \widehat{\mathcal{K}}_{\alpha_{i}^{S}}^{\vec{\mu}}\left(f_{i}\right)$ and $w_{i} \in$ $\widehat{\mathcal{K}}_{\alpha_{i}^{S}}^{\mu}\left(\chi_{\Omega_{i}}\right)$ such that $\sum_{i \in S} v_{i}=\sum_{i \in S} w_{i}$. An $e^{S}$-allocation is simply said to be an $S$-allocation.

Note that $\left(\chi_{A_{1}}, \ldots, \chi_{A_{n}}\right)$ is an $\alpha^{S}$-allocation with $\left(\chi_{E_{i}}, \chi_{F_{i}}\right) \in \widehat{\mathcal{K}}_{\alpha_{i}^{S}}^{\vec{\mu}}\left(\chi_{A_{i}}\right) \times \widehat{\mathcal{K}}_{\alpha_{i}^{S}}^{\vec{\mu}}\left(\chi_{\Omega_{i}}\right)$ for each $i \in S$ if and only if $\left(A_{1}, \ldots, A_{n}\right)$ is an $\alpha^{S}$-partition with $\left(E_{i}, F_{i}\right) \in \mathcal{K}_{\alpha_{i}^{S}}^{\vec{\mu}}\left(A_{i}\right) \times \mathcal{K}_{\alpha_{i}^{S}}^{\vec{\mu}}\left(\Omega_{i}\right)$ for each $i \in S$. Thus, the notion of $\alpha^{S}$-allocations introduced here is a consistent extension of that of $\alpha^{S}$-partitions to $L^{\infty}$-spaces.

Definition 3.4. A fuzzy coalition $\alpha^{S}$ improves upon an allocation $\left(f_{1}, \ldots, f_{n}\right)$ if there exists an $\alpha^{S}$-allocation $\left(g_{1}, \ldots, g_{n}\right)$ such that $\hat{v}_{i}\left(f_{i}\right)<\hat{v}_{i}\left(g_{i}\right)$ for each $i \in S$. An allocation that cannot be improved upon by any fuzzy coalition is a fuzzy core allocation. 
When commodities are homogeneous and divisible as in classical exchange economies, the usual definition of an $\alpha^{S}$-allocation is an allocation $\left(f_{1}, \ldots, f_{n}\right)$ such that:

$$
\sum_{i \in S} \alpha_{i}^{S} f_{i}=\sum_{i \in S} \alpha_{i}^{S} \chi_{\Omega_{i}}
$$

(See $[2,3,12,14]$.) Although the definition of $\alpha^{S}$-allocations in the sense of (3.1) seems to make sense in the extended economy $\widehat{\mathcal{E}}$, it is inadequate in that it cannot be reduced to the corresponding definition of $\alpha^{S}$-partitions in the original economy $\mathcal{E}$.

To illustrate how this definition malfunctions, consider for $n=2$ any fuzzy coalition $\alpha=\left(\alpha_{1}, \alpha_{2}\right) \in[0,1]^{2}$ with $\alpha_{1} \neq \alpha_{2}$. Restricting the definition of $\alpha$-allocations in the sense of (3.1) to the characteristic functions $\left(\chi_{A_{1}}, \chi_{A_{2}}\right)$ with $\chi_{A_{1}}+\chi_{A_{2}}=1$ yields $\alpha_{1}\left(\chi_{A_{1}}-\chi_{\Omega_{1}}\right)=\alpha_{2}\left(\chi_{\Omega_{2}}-\chi_{A_{2}}\right)$, which is true if and only if $A_{1}=\Omega_{1}$ and $A_{2}=\Omega_{2}$. So, every $\alpha$-partition with $\alpha_{1} \neq \alpha_{2}$ in the sense of (3.1) consists of only the initial partition $\left(\Omega_{1}, \Omega_{2}\right)$. Fuzziness entirely disappears from the definition.

\section{Existence of fuzzy core allocations}

\subsection{The NTU game for exchange economies}

Let $\mathcal{N}=2^{N} \backslash\{\emptyset\}$. The market game $V: \mathcal{N} \rightarrow 2^{\mathbb{R}^{n}}$ with NTU for the exchange economy $\widehat{\mathcal{E}}=\left\langle\mathcal{X}, \chi_{\Omega_{i}}, \hat{v}_{i}\right\rangle_{i \in N}$ is given by

$$
V(S)=\left\{\left(x_{1}, \ldots, x_{n}\right) \in \mathbb{R}^{n} \mid \exists \alpha^{S} \text {-allocation }\left(f_{1}, \ldots, f_{n}\right) \text { such that } x_{i} \leq \hat{v}_{i}\left(f_{i}\right), \forall i \in S\right\} .
$$

By construction, $V(S)$ is the utility possibility set of the players (individuals) in $S$ in which payoff vectors are attainable via some fuzzy coalition $\alpha^{S}$. The core $C(V)$ of $V$ is given by ${ }^{1}$

$$
C(V)=\left\{\left(x_{1}, \ldots, x_{n}\right) \in V(N) \mid \nexists(S, y) \in \mathcal{N} \times V(S) \text { such that } x_{i}<y_{i}, \forall i \in S\right\} .
$$

Proposition 4.1. If for an exchange economy $\mathcal{E}=\left\langle(\Omega, \mathcal{F}), \Omega_{i}, v_{i}\right\rangle_{i \in N}$, utility function $v_{i}$ is of the form $v_{i}=\varphi_{i} \circ \vec{\mu}$ such that $\varphi_{i}: \mathcal{R}(\vec{\mu}) \rightarrow \mathbb{R}$ is continuous and quasiconcave for each $i \in N$, then $C(V)$ is nonempty.

Proof. By the celebrated theorem of Scarf [25], the core of the NTU game $V$ is nonempty if $V$ is comprehensive below and balanced, $V(S)$ is closed and bounded from above for every $S \in \mathcal{N}$, and $x=\left(x_{1}, \ldots, x_{n}\right) \in \mathbb{R}^{n}, y=\left(y_{1}, \ldots, y_{n}\right) \in$ $V(S)$ and $x_{i}=y_{i}$ for each $i \in S$ imply $x \in V(S)$. We show that $V$ satisfies these conditions.

It is easy to see that each $V(S)$ is comprehensive from below, i.e., $x=\left(x_{1}, \ldots, x_{n}\right) \in \mathbb{R}^{n}, y=\left(y_{1}, \ldots, y_{n}\right) \in V(S)$ and $x_{i} \leq y_{i}$ for each $i \in N$ imply $x \in V(S)$. Moreover, $x \in \mathbb{R}^{n}, y \in V(S)$ and $x_{i}=y_{i}$ for each $i \in S$ imply $x \in V(S)$. Since each $\hat{v}_{i}$ is weakly* continuous by Lemma 3.1, and hence, bounded on the weakly* compact set $\mathcal{X}$, for each $S \in \mathcal{N}$ there exists $M_{S} \in \mathbb{R}$ such that $x_{i} \leq M_{S}$ for every $x \in V(S)$ and $i \in S$.

We shall show that $V$ is a balanced game. To this end, let $\mathcal{B}$ be a balanced family in $\mathcal{N}$ with balanced weights $\left\{\lambda^{S} \geq 0 \mid S \in \mathcal{B}\right\}$ and let $\mathcal{B}_{i}=\{S \in \mathcal{B} \mid i \in S\}$. We then have $\sum_{S \in \mathcal{B}_{i}} \lambda^{S}=1$ for each $i=1, \ldots, n$. Define:

$$
\chi_{i}^{S}=\left\{\begin{array}{l}
1 \text { if } S \in \mathcal{B}_{i}, \\
0 \text { otherwise }
\end{array} \quad \text { and } t^{S}=\frac{1}{n} \sum_{i \in N} \lambda^{S} \chi_{i}^{S} .\right.
$$

Then, we have:

$$
\sum_{S \in \mathcal{B}} t^{S}=\frac{1}{n} \sum_{S \in \mathcal{B}} \sum_{i \in N} \lambda^{S} \chi_{i}^{S}=\frac{1}{n} \sum_{i \in N} \sum_{S \in \mathcal{B}_{i}} \lambda^{S}=1 .
$$

Choose any $x=\left(x_{1}, \ldots, x_{n}\right) \in \bigcap_{S \in \mathcal{B}} V(S)$. Then, for every $S \in \mathcal{B}$, there exists an $\alpha^{S}$-allocation $\left(f_{1}^{S}, \ldots, f_{n}^{S}\right)$ such that $x_{i} \leq \hat{v}_{i}\left(f_{i}^{S}\right)$ for each $i \in S$. Let $f_{i}=\sum_{S \in \mathcal{B}} t^{S} f_{i}^{S}$ for each $i \in N$. Then, $\left(f_{1}, \ldots, f_{n}\right)$ is an allocation because $\mathcal{A}$

\footnotetext{
${ }^{1}$ This definition of the core is sometimes called the weak core in the literature.
} 
is convex by Lemma 3.2. Since $x_{i} \leq \hat{v}_{i}\left(f_{i}\right)$ for each $i \in N$ by the quasiconcavity of $\hat{v}_{i}$ established in Lemma 3.1, we have $x \in V(N)$. Therefore, $\bigcap_{S \in \mathcal{B}} V(S) \subset V(N)$, and consequently, $V$ is balanced.

We next show that $V(S)$ is closed for every $S \in \mathcal{B}$. Let $\left\{x^{q}\right\}$ be a sequence in $V(S)$ converging to $x \in \mathbb{R}^{n}$. Then, there exists an allocation $\left(f_{1}^{q}, \ldots, f_{n}^{q}\right)$ such that $x_{i}^{q} \leq \hat{v}_{i}\left(f_{i}^{q}\right)$ for each $i \in S$ and $q=1,2, \ldots$ Since $\mathcal{A}$ is weakly* compact by Lemma 3.2, the sequence $\left\{\left(f_{1}^{q}, \ldots, f_{n}^{q}\right)\right\}$ contains a subsequence that is weakly* convergent to $\left(f_{1}, \ldots, f_{n}\right) \in \mathcal{A}$. Then we have $x_{i} \leq \hat{v}_{i}\left(f_{i}\right)$ for each $i \in S$ by the weak* continuity of $\hat{v}_{i}$. It is easy to verify that $\left(f_{1}, \ldots, f_{n}\right)$ is an $\alpha^{S}$-allocation. Thus, we obtain $x \in V(S)$, and hence, $V(S)$ is closed.

Corollary 4.1. If for an exchange economy $\mathcal{E}=\left\langle(\Omega, \mathcal{F}), \Omega_{i}, v_{i}\right\rangle_{i \in N}$, utility function $v_{i}$ is of the form $v_{i}=\varphi_{i} \circ \vec{\mu}$ such that $\varphi_{i}: \mathcal{R}(\vec{\mu}) \rightarrow \mathbb{R}$ is continuous and quasiconcave for each $i \in N$, then there exists a fuzzy core allocation for $\widehat{\mathcal{E}}=\left\langle\mathcal{X}, \chi_{\Omega_{i}}, \hat{v}_{i}\right\rangle_{i \in N}$.

Proof. By Proposition 4.1, one can choose an element $\left(x_{1}, \ldots, x_{n}\right)$ in $C(V)$. Then there exists an $\alpha^{N}$-allocation $\left(f_{1}, \ldots, f_{n}\right)$ such that $x_{i} \leq \hat{v}_{i}\left(f_{i}\right)$ for each $i \in N$. Suppose that $\left(f_{1}, \ldots, f_{n}\right)$ is not a fuzzy core allocation. Then there exists an $\alpha^{S}$-allocation $\left(g_{1}, \ldots, g_{n}\right)$ such that $\hat{v}_{i}\left(f_{i}\right)<\hat{v}_{i}\left(g_{i}\right)$ for each $i \in S$. Then we have $\left(\hat{v}_{1}\left(g_{1}\right), \ldots, \hat{v}_{n}\left(g_{n}\right)\right) \in V(S)$ and $x_{i}<\hat{v}_{i}\left(g_{i}\right)$ for each $i \in S$, which contradicts the fact that $\left(x_{1}, \ldots, x_{n}\right)$ is in $C(V)$.

\subsection{Existence of fuzzy core partitions}

Let $x=\left(x_{1}, \ldots, x_{n}\right)$ be in $C(V)$ and define the set $\mathcal{C}_{x}$ by

$$
\mathcal{C}_{x}=\left\{\left(f_{1}, \ldots, f_{n}\right) \in \mathcal{A} \mid x_{i} \leq \hat{v}_{i}\left(f_{i}\right), \forall i \in N\right\}
$$

It is easy to verify that $\mathcal{C}_{x}$ is a subset of the set of fuzzy core allocations for $\widehat{\mathcal{E}}=\left\langle\mathcal{X}, \chi_{\Omega_{i}}, \hat{v}_{i}\right\rangle_{i \in N}$.

Proposition 4.2. If for an exchange economy $\mathcal{E}=\left\langle(\Omega, \mathcal{F}), \Omega_{i}, v_{i}\right\rangle_{i \in N}$, utility function $v_{i}$ is of the form $v_{i}=\varphi_{i} \circ \vec{\mu}$ such that $\varphi_{i}: \mathcal{R}(\vec{\mu}) \rightarrow \mathbb{R}$ is continuous and quasiconcave for each $i \in N$, then there exists a partition $\left(A_{1}, \ldots, A_{n}\right)$ of $\Omega$ such that $\left(\chi_{A_{1}}, \ldots, \chi_{A_{n}}\right) \in \mathcal{C}_{x}$.

The proof of the proposition is essentially based on the ingenious technique of Lindenstrauss [18], yielding that an extreme point of $\mathcal{C}_{x}$ is indeed a measurable partition of $\Omega$ (see also [1]).

Let $K$ be a nonempty subset of a Banach space $X$. A point $f \in K$ is an extreme point of $K$ if it is not a proper convex combination of two points in $K$, i.e., $f=\alpha f_{0}+(1-\alpha) f_{1}$ with $f_{1}, f_{2} \in K$ and $\alpha \in(0,1)$ implies $f_{0}=f_{1}$.

The following result is a special case of the Krein-Milman theorem (see [8, Lemma V.8.2]).

\section{Theorem 4.1 (Krein-Milman). A nonempty weakly* compact subset of a Banach space has extreme points.}

Proof of Proposition 4.2. Note that $\mathcal{C}_{x}$ is nonempty, convex and weakly* compact by Proposition 4.1, and Lemmas 3.1 and 3.2. According to the Krein-Milman theorem, $\mathcal{C}_{x}$ has an extreme point $\left(f_{1}, \ldots, f_{n}\right)$. We shall show that each of $f_{i}$ is a characteristic function. Suppose to the contrary, that $f_{i}$ is not a characteristic function for some $i \in N$. By virtue of that $\left(f_{1}, \ldots, f_{n}\right) \in \mathcal{A}$, we may assume without loss of generality that there exist $\varepsilon>0$ and $A \in \mathcal{F}$ with $\mu(A)>0$ such that $\varepsilon<f_{1}, f_{2}<1-\varepsilon$ on $A$. It follows from the Lyapunov's convexity theorem that there exists a measurable subset $B \subset A$ such that $\vec{\mu}(B)=\frac{1}{2} \vec{\mu}(A)$. Set $h=\varepsilon\left(\chi_{A}-2 \chi_{B}\right)$. Then $h \neq 0,0 \leq f_{1} \pm h, f_{2} \pm h \leq 1$, and $\vec{\mu}(h)=0$. Since $\hat{v}_{i}\left(f_{i} \pm h\right)=\varphi_{i}\left(\vec{\mu}\left(f_{i} \pm h\right)\right)=\hat{v}_{i}\left(f_{i}\right) \geq x_{i}$ for $i=1,2$, we have $\left(f_{1} \pm h, f_{2} \mp h, f_{3}, \ldots, f_{n}\right) \in \mathcal{C}_{x}$. This yields:

$$
\left(f_{1}, \ldots, f_{n}\right)=\frac{1}{2}\left[\left(f_{1}+h, f_{2}-h, f_{3}, \ldots, f_{n}\right)+\left(f_{1}-h, f_{2}+h, f_{3}, \ldots, f_{n}\right)\right] \in \mathcal{C}_{x},
$$

which means that $\left(f_{1}, \ldots, f_{n}\right)$ is a convex combination of the distinct elements $\left(f_{1}+h, f_{2}-h, f_{3}, \ldots, f_{n}\right)$ and $\left(f_{1}-h, f_{2}+h, f_{3}, \ldots, f_{n}\right)$ in $\mathcal{C}_{x}$, a contradiction to the fact that $\left(f_{1}, \ldots, f_{n}\right)$ is an extreme point in $\mathcal{C}_{x}$.

The next theorem is now an immediate consequence of Proposition 4.2 and Corollary 4.1. 
Theorem 4.2. If for an exchange economy $\mathcal{E}=\left\langle(\Omega, \mathcal{F}), \Omega_{i}, v_{i}\right\rangle_{i \in N}$, utility function $v_{i}$ is of the form $v_{i}=\varphi_{i} \circ \vec{\mu}$ such that $\varphi_{i}: \mathcal{R}(\vec{\mu}) \rightarrow \mathbb{R}$ is continuous and quasiconcave for each $i \in N$, then there exists a fuzzy core partition.

Corollary 4.2. If for an exchange economy $\mathcal{E}=\left\langle(\Omega, \mathcal{F}), \Omega_{i}, v_{i}\right\rangle_{i \in N}$, utility function $v_{i}$ is $\vec{\mu}$-concave and $\vec{\mu}$-continuous at every $A \in \mathcal{F}$ with $\vec{\mu}(A) \in$ bd $\mathcal{R}(\vec{\mu})$ for each $i \in N$, then there exists a fuzzy core partition.

Proof. By Corollary 2.1, $v_{i}$ is of the form $v_{i}=\varphi_{i} \circ \vec{\mu}$ such that $\varphi_{i}: \mathcal{R}(\vec{\mu}) \rightarrow \mathbb{R}$ is continuous and concave for each $i \in N$. The corollary follows from Theorem 4.2.

Since a fuzzy core partition is a core partition, Theorem 4.2 is an extension of Hüsseinov [15] and Sagara and Vlach [24], the former proved the existence of core partitions for the case, where utility functions of the individuals are continuous quasiconcave transformations of a finite-dimensional nonatomic vector measure and the latter for the case of concave measures.

\section{Optimality and supporting prices}

In this section we characterize (weak) Pareto optimal allocations for an extended exchange economy $\widehat{\mathcal{E}}=\left\langle\mathcal{X}, \chi_{\Omega_{i}}\right.$, $\left.\hat{v}_{i}\right\rangle_{i \in N}$ in terms of supporting prices. Under the continuity, quasiconcavity and strict monotonicity assumptions on utility functions, the supportability of allocations by prices is shown to be equivalent to Pareto optimality. This observation leads to the second fundamental theorem of welfare economics for the present model: every Pareto optimal allocation is a competitive equilibrium with transfers of economy $\mathcal{E}=\left\langle(\Omega, \mathcal{F}), \Omega_{i}, v_{i}\right\rangle_{i \in N}$. For the existence of competitive equilibria for $\mathcal{E}=\left\langle(\Omega, \mathcal{F}), \Omega_{i}, v_{i}\right\rangle_{i \in N}$ in a more general setting, see Hüsseinov [17].

\subsection{Existence of supporting prices}

Let $b a(\Omega, \mathcal{F}, \mu)$ be the vector subspace of $b a(\Omega, \mathcal{F})$ whose elements vanish at every $A \in \mathcal{F}$ with $\mu(A)=0$. Then, $b a(\Omega, \mathcal{F}, \mu)$ is the dual space of $L^{\infty}(\Omega, \mathcal{F}, \mu)$ (see [8, Theorem IV.8.16]).

Definition 5.1. A nonzero element $\pi \in b a(\Omega, \mathcal{F}, \mu)$ is a supporting price for an allocation $\left(f_{1}, \ldots, f_{n}\right)$ for $\widehat{\mathcal{E}}=$ $\left\langle\mathcal{X}, \chi_{\Omega_{i}}, \hat{v}_{i}\right\rangle_{i \in N}$ if $\hat{v}_{i}\left(f_{i}\right) \leq \hat{v}_{i}(f)$ for $f \in \mathcal{X}$ implies $\pi\left(f_{i}\right) \leq \pi(f)$.

Definition 5.2. An allocation $\left(f_{1}, \ldots, f_{n}\right)$ for $\widehat{\mathcal{E}}=\left\langle\mathcal{X}, \chi_{\Omega_{i}}, \hat{v}_{i}\right\rangle_{i \in N}$ is

(i) weakly Pareto optimal if there exists no allocation $\left(g_{1}, \ldots, g_{n}\right)$ such that $\hat{v}_{i}\left(f_{i}\right)<\hat{v}_{i}\left(g_{i}\right)$ for each $i \in N$.

(ii) Pareto optimal if there exists no allocation $\left(g_{1}, \ldots, g_{n}\right)$ such that $\hat{v}_{i}\left(f_{i}\right) \leq \hat{v}_{i}\left(g_{i}\right)$ for each $i \in N$ and $\hat{v}_{j}\left(f_{j}\right)<\hat{v}_{j}\left(g_{j}\right)$ for some $j \in N$.

It is easy to see that every (fuzzy) core allocation for $\widehat{\mathcal{E}}=\left\langle\mathcal{X}, \chi_{\Omega_{i}}, \hat{v}_{i}\right\rangle_{i \in N}$ is weakly Pareto optimal. As shown below, Pareto optimality and weak Pareto optimality coincide whenever each $\varphi_{i}$ satisfies continuity.

A function $\varphi: \mathcal{R}(\vec{\mu}) \rightarrow \mathbb{R}$ is strictly monotonic if $x=\left(x_{1}, \ldots, x_{m}\right), y=\left(y_{1}, \ldots, y_{m}\right) \in \mathcal{R}(\vec{\mu})$ and $x_{k}<y_{k}$ for each $k=1, \ldots, m$ imply $\varphi(x)<\varphi(y)$.

Proposition 5.1. If for an exchange economy $\mathcal{E}=\left\langle(\Omega, \mathcal{F}), \Omega_{i}, v_{i}\right\rangle_{i \in N}$, utility function $v_{i}$ is of the form $v_{i}=\varphi_{i} \circ \vec{\mu}$ such that $\varphi_{i}: \mathcal{R}(\vec{\mu}) \rightarrow \mathbb{R}$ is continuous and strictly monotonic for each $i \in N$, then an allocation for $\widehat{\mathcal{E}}=\left\langle\mathcal{X}, \chi_{\Omega_{i}}, \hat{v}_{i}\right\rangle_{i \in N}$ is Pareto optimal if and only if it is weakly Pareto optimal.

Proof. It is evident that Pareto optimality implies weak Pareto optimality. We show the converse implication. Let $\left(f_{1}, \ldots, f_{n}\right)$ be an allocation for $\widehat{\mathcal{E}}$ that is not Pareto optimal. Then, there is an allocation $\left(g_{1}, \ldots, g_{n}\right)$ such that $\hat{v}_{i}\left(f_{i}\right) \leq \hat{v}_{i}\left(g_{i}\right)$ for each $i \in N$ and $\hat{v}_{j}\left(f_{j}\right)<\hat{v}_{j}\left(g_{j}\right)$ for some $j \in N$. As $\varphi_{j}$ is strictly monotonic, there exists $A \in \mathcal{F}$ with $\mu(A)>0$ on which $g_{j}$ is positive. The mutual absolute continuity of $\mu_{1}, \ldots, \mu_{m}$ yields $\mu_{k}(A)>0$ for each $k=$ $1, \ldots, m$. By the weak* continuity of $\hat{v}_{j}$ established in Lemma 3.1, there is $\varepsilon \in(0,1)$ such that $\hat{v}_{j}\left(f_{j}\right)<\hat{v}_{j}\left((1-\varepsilon) g_{j}\right)$. 
Define $h_{i} \in L^{\infty}(\Omega, \mathcal{F}, \mu)$ by

$$
h_{i}= \begin{cases}g_{i}+\frac{\varepsilon}{n-1} g_{j} & \text { if } i \neq j, \\ (1-\varepsilon) g_{j} & \text { otherwise }\end{cases}
$$

It is easy to see that $0 \leq h_{i} \leq 1$ for each $i \in N, h_{i} \geq g_{i}$ and $\mu_{k}\left(h_{i}\right)=\mu_{k}\left(g_{i}\right)+\varepsilon \mu_{k}\left(g_{j}\right) /(n-1)>\mu_{k}\left(g_{i}\right)$ for $i \neq j$ and $k=1, \ldots, m$. By the strict monotonicity of $\varphi_{i}$, the resulting allocation $\left(h_{1}, \ldots, h_{n}\right)$ satisfies $\hat{v}_{i}\left(f_{i}\right)<\hat{v}_{i}\left(h_{i}\right)$ for each $i \in N$. Thus, allocation $\left(f_{1}, \ldots, f_{n}\right)$ is not weakly Pareto optimal.

A nonzero element $\pi \in b a(\Omega, \mathcal{F})$ is positive if $\pi(A) \geq 0$ for every $A \in \mathcal{F}$.

Theorem 5.1. If for an exchange economy $\mathcal{E}=\left\langle(\Omega, \mathcal{F}), \Omega_{i}, v_{i}\right\rangle_{i \in N}$, utility function $v_{i}$ is of the form $v_{i}=\varphi_{i} \circ \vec{\mu}$ such that $\varphi_{i}: \mathcal{R}(\vec{\mu}) \rightarrow \mathbb{R}$ is continuous, quasiconcave and strictly monotonic for each $i \in N$, then an allocation for $\widehat{\mathcal{E}}=\left\langle\mathcal{X}, \chi_{\Omega_{i}}, \hat{v}_{i}\right\rangle_{i \in N}$ is Pareto optimal if and only if it has a positive supporting price.

To prove the theorem, we need the following lemma, whose proof is postponed to Appendix.

Lemma 5.1. If $\varphi: \mathcal{R}(\vec{\mu}) \rightarrow \mathbb{R}$ is continuous, quasiconcave and strictly monotonic, then $\varphi$ has an extension $\hat{\varphi}: \mathbb{R}_{+}^{m} \rightarrow$ $\mathbb{R}$ preserving its properties.

Let $\widehat{\mathcal{E}}=\left\langle\mathcal{X}, \chi_{\Omega_{i}}, \hat{v}_{i}\right\rangle_{i \in N}$ be an extended economy where $\hat{v}_{i}=\varphi_{i} \circ \vec{\mu}$ is continuous, quasiconcave and strictly monotone for each $i \in N$. Then, each utility function $\hat{v}_{i}$ can be extended further from $\mathcal{X}$ to the positive cone $L_{+}^{\infty}(\Omega, \mathcal{F}, \mu)$ of $L^{\infty}(\Omega, \mathcal{F}, \mu)$. To this end, let $\hat{\varphi}_{i}: \mathbb{R}_{+}^{m} \rightarrow \mathbb{R}$ be a continuous, quasiconcave, strictly monotonic extension of $\varphi_{i}:$ $\mathcal{R}(\vec{\mu}) \rightarrow \mathbb{R}$ provided by Lemma 5.1. Then, we obtain an extension $\hat{\hat{v}}_{i}: L_{+}^{\infty}(\Omega, \mathcal{F}, \mu) \rightarrow \mathbb{R}$ of $v_{i}$ by $\hat{\hat{v}}_{i}=\hat{\varphi}_{i} \circ \vec{\mu}$. We denote by $\widehat{\mathcal{E}}=\left\langle L_{+}^{\infty}(\Omega, \mathcal{F}, \mu), \chi_{\Omega_{i}}, \hat{\hat{v}}_{i}\right\rangle_{i \in N}$ the extension of the economy $\widehat{\mathcal{E}}$. Allocations for $\widehat{\mathcal{E}}$ are precisely those for $\widehat{\mathcal{E}}$ and supporting prices for $\widehat{\mathcal{E}}$ are those for $\widehat{\mathcal{E}}$. This observation is employed in the sequel.

Proof of Theorem 5.1. Assume that an allocation $\left(f_{1}, \ldots, f_{n}\right)$ for an extended economy $\widehat{\mathcal{E}}=\left\langle\mathcal{X}, \chi_{\Omega_{i}}, \hat{v}_{i}\right\rangle_{i \in N}$ has a positive supporting price $\pi \in b a(\Omega, \mathcal{F}, \mu)$. Also, suppose by way of contradiction that $\left(f_{1}, \ldots, f_{n}\right)$ is not Pareto optimal. Then by Proposition 5.1, there exists another allocation $\left(g_{1}, \ldots, g_{n}\right)$ for $\widehat{\mathcal{E}}$ such that $\hat{v}_{i}\left(f_{i}\right)<\hat{v}_{i}\left(g_{i}\right)$ for each $i \in N$. By the supportability of $\pi$, we have $\pi\left(f_{i}\right) \leq \pi\left(g_{i}\right)$ for each $i \in N$. Since $0<\pi(\Omega)=\sum_{i \in N} \pi\left(f_{i}\right)=$ $\sum_{i \in N} \pi\left(g_{i}\right)$, we must have $\pi\left(f_{i}\right)=\pi\left(g_{i}\right)$ for each $i \in N$. By the weak* continuity of $\hat{v}_{i}$ established in Lemma 3.1, there is $\varepsilon \in(0,1)$ such that $\hat{v}_{i}\left(f_{i}\right)<\hat{v}_{i}\left(\varepsilon g_{i}\right)$ for each $i \in N$. Again, by the supportability of $\pi$, we obtain $\pi\left(f_{i}\right) \leq \varepsilon \pi\left(g_{i}\right)=\varepsilon \pi\left(f_{i}\right)$, which yields $\pi\left(f_{i}\right)=0$ for each $i \in N$, and hence, $0=\sum_{i \in N} \pi\left(f_{i}\right)=\pi(\Omega)$, a contradiction.

Conversely, let $\left(f_{1}, \ldots, f_{n}\right)$ be a Pareto optimal allocation for $\widehat{\mathcal{E}}$. Then, it is likewise a Pareto optimal allocation for the extended economy $\widehat{\mathcal{E}}$. Let int $L_{+}^{\infty}(\Omega, \mathcal{F}, \mu)$ be the norm interior of $L_{+}^{\infty}(\Omega, \mathcal{F}, \mu)$. Define $G_{i}=\{g \in$ $\left.L_{+}^{\infty}(\Omega, \mathcal{F}, \mu) \mid \hat{\hat{v}}_{i}\left(f_{i}\right) \leq \hat{\hat{v}}_{i}(g)\right\}$ for each $i \in N$ and $G=\sum_{i \in N} G_{i}-1$. Since $\hat{\varphi}_{i}$ is quasiconcave, $G_{i}$ is convex, and hence, $G$ is also convex. We shall show that $G \cap\left(-\operatorname{int} L_{+}^{\infty}(\Omega, \mathcal{F}, \mu)\right)=\emptyset$. Suppose, to the contrary, that the intersection $G \cap\left(-\right.$ int $\left.L_{+}^{\infty}(\Omega, \mathcal{F}, \mu)\right)$ is nonempty. Then, there exists $g_{i} \in G_{i}$ and $v \in \operatorname{int} L_{+}^{\infty}(\Omega, \mathcal{F}, \mu)$ such that $\sum_{i \in N} g_{i}+v=1$. Setting $h_{i}=g_{i}+(1 / n) v$ for each $i \in N$ yields an allocation $\left(h_{1}, \ldots, h_{n}\right)$ such that $\hat{\hat{v}}_{i}\left(f_{i}\right)<\hat{\hat{v}}_{i}\left(h_{i}\right)$ for each $i \in N$ in view of $v>0$ and the strict monotonicity of $\hat{\varphi}_{i}$ proven in Lemma 5.1. This contradicts the (weak) Pareto optimality of $\left(f_{1}, \ldots, f_{n}\right)$.

It follows from the separation theorem (see [8, Theorem V.1.12]) that there exist a nonzero element $\pi \in b a(\Omega, \mathcal{F}, \mu)$ and a constant $a \in \mathbb{R}$ such that $-\pi(f) \leq a \leq \pi(g)$ for every $f \in \operatorname{int} L_{+}^{\infty}(\Omega, \mathcal{F}, \mu)$ and $g \in G$. Since $0 \in G$, we have $a \leq 0$. If $a<0$, then $\pi(f) \geq-a>0$ for every $f \in \operatorname{int} L_{+}^{\infty}(\Omega, \mathcal{F}, \mu)$. However, since $\pi(0)=0$ and $\pi$ is continuous at the origin in the norm topology of $L^{\infty}(\Omega, \mathcal{F}, \mu)$, there exists $\delta>0$ such that $|\pi(f)|<-a$ for every $f \in L^{\infty}(\Omega, \mathcal{F}, \mu)$ with $\|f\|_{\infty}<\delta$, a contradiction. Hence, $a=0$. We claim that $\pi$ is positive. Suppose to the contrary, that $\pi(A)<0$ for some $A \in \mathcal{F}$. Let $f \in$ int $L_{+}^{\infty}(\Omega, \mathcal{F}, \mu)$ be arbitrary and take $\varepsilon>0$ sufficiently small so that $\pi(A)+\varepsilon \pi(f)<0$. Then, we have $\pi\left(\chi_{A}+\varepsilon f\right)=\pi(A)+\varepsilon \pi(f)<0$, a contradiction, because $\chi_{A}+\varepsilon f \in \operatorname{int} L_{+}^{\infty}(\Omega, \mathcal{F}, \mu)$. 
If $\hat{\hat{v}}_{i}\left(f_{i}\right) \leq \hat{\hat{v}}_{i}(g)$, then $g-f_{i} \in G$ because $g-f_{i} \in G_{i}-\left(1-\sum_{j \in N \backslash\{i\}} f_{j}\right) \subset \sum_{i \in N} G_{i}-1=G$. By the separation property in the above, $\pi\left(f_{i}\right) \leq \pi(g)$ must hold. Therefore, $\pi$ is a nonnegative supporting price for $\left(f_{1}, \ldots, f_{n}\right)$ for $\widehat{\hat{\mathcal{E}}}$.

\subsection{Supporting prices in $L^{1}$}

As observed by Bewley [5, p. 516], "one could call any element of $b a$ a price system, but since those elements of $b a$ not belonging to $L^{1}$ have no economic interpretation, we will be interested only in equilibria with price systems in $L^{1}$." "[T]heorem [5.1] ... would be of little interest if one could not find interesting conditions under which equilibrium price systems could be chosen from $L^{1}$." (See [5, p. 523].)

Theorem 5.2. If for an exchange economy $\mathcal{E}=\left\langle(\Omega, \mathcal{F}), \Omega_{i}, v_{i}\right\rangle_{i \in N}$, utility function $v_{i}$ is of the form $v_{i}=\varphi_{i} \circ \vec{\mu}$ such that $\varphi_{i}: \mathcal{R}(\vec{\mu}) \rightarrow \mathbb{R}$ is continuous, quasiconcave and strictly monotonic for each $i \in N$, then every Pareto optimal allocation (in particular, every fuzzy core partition) for $\widehat{\mathcal{E}}=\left\langle\mathcal{X}, \chi_{\Omega_{i}}, \hat{v}_{i}\right\rangle_{i \in N}$ has a positive supporting price in $L^{1}(\Omega, \mathcal{F}, \mu)$.

To prove the theorem, the Yosida-Hewitt decomposition of finitely additive measures (see [26, Theorems 1.22 and 1.24, 8, Theorem III.7.6.8, 8, Theorem III.7.6.8]) plays a crucial role.

A positive element $\pi \in b a(\Omega, \mathcal{F})$ is purely finitely additive if every countably additive measure $\lambda$ satisfying $0 \leq \lambda \leq \pi$ is identically zero.

\section{Theorem 5.3 (Yosida and Hewitt).}

(i) A positive element $\pi \in b a(\Omega, \mathcal{F})$ is decomposed uniquely into $\pi=\pi^{\mathrm{c}}+\pi^{\mathrm{p}}$, where $\pi^{\mathrm{c}} \geq 0$ is countably additive and $\pi^{\mathrm{p}} \geq 0$ is purely finitely additive.

(ii) If $\pi \in b a(\Omega, \mathcal{F})$ is purely finitely additive and $\pi \geq 0$, and $\lambda \geq 0$ is countably additive, then there exists a sequence $\left\{A^{q}\right\}$ in $\mathcal{F}$ such that:
(a) $A^{q} \subset A^{q+1}$ for each $q=1,2, \ldots$;
(b) $\lim _{q} \lambda\left(\Omega \backslash A^{q}\right)=0$;
(c) $\pi\left(A^{q}\right)=0$ for each $q=1,2, \ldots$..

Proof of Theorem 5.2. Let $\pi \in b a(\Omega, \mathcal{F}, \mu)$ be a positive supporting price for a weakly Pareto optimal allocation $\left(f_{1}, \ldots, f_{n}\right)$ for $\widehat{\mathcal{E}}$, whose existence is assured in Theorem 5.1. It follows from Theorem 5.3(i) that $\pi$ is decomposed uniquely into the countable additive part $\pi^{\mathrm{c}} \geq 0$ and the purely finitely additive part $\pi^{\mathrm{p}} \geq 0$ such that $\pi=\pi^{\mathrm{c}}+\pi^{\mathrm{p}}$. Moreover, by Theorem 5.3(ii), there exists a sequence $\left\{A^{q}\right\}$ in $\mathcal{F}$ such that (a) $A^{q} \subset A^{q+1}$ for each $q=1,2, \ldots ;$ (b) $\lim _{q} \mu\left(\Omega \backslash A^{q}\right)=0$; (c) $\pi^{\mathrm{p}}\left(f \chi_{A^{q}}\right)=0$ for every $f \in L^{\infty}(\mathcal{F}, \Omega, \mu)$ and $q=1,2, \ldots$

First we show that $\pi^{\mathrm{c}}$ is nonzero. Suppose to the contrary that $\pi^{\mathrm{c}}=0$. Since $\sum_{i \in N} \pi\left(f_{i}\right)=\pi(\Omega)>0$, there exists $j \in N$ such that $\pi\left(f_{j}\right)>0$. Take any function $g \in L^{1}(\Omega, \mathcal{F}, \mu)$. Then, we have

$$
\left|\int f_{j} \chi_{A^{q}} g d \mu-\int f_{j} g d \mu\right| \leq\left\|f_{j}\right\|_{\infty} \int\left|1-\chi_{A^{q}}\right||g| d \mu=\left\|f_{j}\right\|_{\infty} \int_{\Omega \backslash A^{q}}|g| d \mu \rightarrow 0
$$

as $q \rightarrow \infty$. Hence, $f_{j} \chi_{A^{q}}$ is weakly* convergent to $f_{j}$ in $L^{\infty}(\Omega, \mathcal{F}, \mu) .{ }^{2}$ Let $\varepsilon>0$ be arbitrary. Since $f_{j} \chi_{A^{q}}+\varepsilon \chi_{\Omega} \rightarrow$ $f+\varepsilon \chi_{\Omega}$ in the weak* topology of $L^{\infty}(\Omega, \mathcal{F}, \mu)$ as $q \rightarrow \infty$, by the weak* continuity of $\hat{\hat{v}}_{j}$ and the strict monotonicity of $\hat{\varphi}_{j}$, we have $\hat{\hat{v}}_{j}\left(f_{j}\right)<\hat{\hat{v}}_{j}\left(f_{j} \chi_{A^{q}}+\varepsilon \chi_{\Omega}\right)$ for all sufficiently large $q$. By the supportability of $\pi$ for $\widehat{\mathcal{E}}$, we have

$$
0<\pi\left(f_{j}\right) \leq \pi\left(f_{j} \chi_{A^{q}}+\varepsilon \chi_{\Omega}\right)=\pi^{\mathrm{c}}\left(f_{j} \chi_{A^{q}}\right)+\pi^{\mathrm{p}}\left(f_{j} \chi_{A^{q}}\right)+\varepsilon \pi(\Omega)=\varepsilon \pi(\Omega) .
$$

Since $\varepsilon>0$ was arbitrary, we obtain $0<\pi\left(f_{j}\right) \leq 0$, a contradiction.

Suppose that $\hat{v}_{i}\left(f_{i}\right) \leq \hat{v}_{i}(f)$. Take any $\varepsilon>0$. Since $f \chi_{A^{q}}+\varepsilon \chi_{\Omega} \rightarrow f+\varepsilon \chi_{\Omega}$ in the weak* topology of $L^{\infty}(\Omega, \mathcal{F}, \mu)$ as $q \rightarrow \infty$, by the weak* continuity of $\hat{\hat{v}}_{i}$ and the strict monotonicity of $\hat{\varphi}_{i}$, we have $\hat{\hat{v}}_{i}\left(f_{i}\right)<\hat{\hat{v}}_{i}\left(f \chi_{A^{q}}+\varepsilon \chi_{\Omega}\right)$ for all

\footnotetext{
${ }^{2}$ More specifically, one can show that this convergence is in the Mackey topology on $L^{\infty}(\Omega, \mathcal{F}, \mu)$. See Bewley [5, Appendix I (24)].
} 
sufficiently large $q$. Since $\pi$ is a supporting price for allocation $\left(f_{1}, \ldots, f_{n}\right)$ for $\widehat{\mathcal{E}}$, we have $\pi\left(f_{i}\right) \leq \pi\left(f \chi_{A^{q}}+\varepsilon \chi_{\Omega}\right)$, and hence

$$
\pi^{\mathrm{c}}\left(f_{i}\right) \leq \pi\left(f_{i}\right) \leq \pi^{\mathrm{c}}\left(f \chi_{A^{q}}+\varepsilon \chi_{\Omega}\right)+\pi^{\mathrm{p}}\left(f \chi_{A^{q}}+\varepsilon \chi_{\Omega}\right)=\pi^{\mathrm{c}}\left(f \chi_{A^{q}}\right)+\varepsilon \pi(\Omega) \leq \pi^{\mathrm{c}}(f)+\varepsilon \pi(\Omega),
$$

where the last inequality employs the fact that $0 \leq f \chi_{A^{q}} \leq f$ and $\pi^{\mathrm{c}} \geq 0$. Since $\varepsilon>0$ was arbitrary, we obtain $\pi^{\mathrm{c}}\left(f_{i}\right) \leq \pi^{\mathrm{c}}(f)$. Therefore, $\pi^{\mathrm{c}}$ is a positive supporting price in $L^{1}(\Omega, \mathcal{F}, \mu)$.

The above proof demonstrates that a supporting price $\pi$ is decomposed uniquely into a countably additive part $\pi^{\mathrm{c}}$ and a purely finitely additive part $\pi^{\mathrm{p}}$, and $\pi^{\mathrm{p}}$ is negligible in the valuation of the Pareto optimal allocation. The supporting price $\pi^{\mathrm{c}}$ has the Radon-Nikodym derivative with respect to $\mu$. Therefore, $\pi^{\mathrm{c}}$ is represented by a state-dependent price $p: \Omega \rightarrow \mathbb{R}$ in $L^{1}(\Omega, \mathcal{F}, \mu)$, where the value of a piece $A \in \mathcal{F}$ is calculated via the integral $\pi^{\mathfrak{c}}(A)=\int_{A} p d \mu$.

"Such prices have very natural economic interpretations. For instance, if we interpret elements of $\Omega$ as representing states of the world, so that a function in $L^{\infty}(\Omega, \mathcal{F}, \mu)$ represents a bundle of contingent commodities, then a function in $L^{1}(\Omega, \mathcal{F}, \mu)$ represents commodity/state prices. However, prices in $b a(\Omega, \mathcal{F}, \mu)$ that do not belong to $L^{1}(\Omega, \mathcal{F}, \mu)$ seem to have no natural economic interpretation." (See [20, p. 1861].)

\section{Acknowledgments}

The first-named author contributed to this paper while visiting the Faculty of Economics, Hosei University. This research is supported by Grants-in-Aid for Scientific Research (No. 21530277 and No. 23530230) from the Ministry of Education, Culture, Sports, Science and Technology, Japan. The authors are grateful for helpful suggestions from an anonymous referee.

\section{Appendix A. Continuous, quasiconcave, strictly monotonic extensions}

For vectors $x=\left(x_{1}, \ldots, x_{n}\right), y=\left(y_{1}, \ldots, y_{n}\right) \in \mathbb{R}^{m}$, denote $x \leq y$ to mean that $x_{k} \leq y_{k}$ for each $k=1, \ldots, m$ and $x \ll y$ to mean that $x_{k}<y_{k}$ for each $k=1, \ldots, m$. The positive and strictly positive orthants of $\mathbb{R}^{m}$ are given, respectively by $\mathbb{R}_{+}^{m}=\left\{x \in \mathbb{R}^{m} \mid x \geq 0\right\}$ and $\mathbb{R}_{++}^{m}=\left\{x \in \mathbb{R}^{m} \mid x \gg 0\right\}$.

Let $C \subset \mathbb{R}^{m}$. A function $\varphi: C \rightarrow \mathbb{R}$ is monotonic if $x \leq y$ and $x, y \in C$ imply $\varphi(x) \leq \varphi(y) ; \varphi$ is strictly monotonic if $x \ll y$ and $x, y \in C$ imply $\varphi(x)<\varphi(y)$.

Proposition A.1. Let $c=\left(c_{1}, \ldots, c_{m}\right) \in \mathbb{R}_{++}^{m}$ and $C$ be a compact convex subset of $\prod_{k=1}^{m}\left[0, c_{k}\right]$ that contains $\mathbf{0}$ and $c$ satisfying $x \ll c$ for every $x \in C$ with $x \neq c$. If $\varphi: C \rightarrow \mathbb{R}$ is continuous, quasiconcave and strictly monotonic, then $\varphi$ has an extension $\hat{\varphi}: \mathbb{R}_{+}^{m} \rightarrow \mathbb{R}$ preserving its properties.

Proof. First, we extend $\varphi$ to $D=\mathbb{R}_{+}^{m} \backslash\left(c+\mathbb{R}_{++}^{m}\right)$. For a point $x \in D$, set $\varphi_{0}(x)=\max \{\varphi(y) \mid y \in C, y \leq x\}$. Then, $\varphi_{0}$ is a continuous extension of $\varphi$ to $D$. To verify this claim, define the set-valued mapping $\Phi: D \rightrightarrows C$ by $\Phi(x)=$ $\{y \in C \mid y \leq x\}$. Then, $\Phi$ is nonempty-, compact-, convex-valued and continuous. Since $\varphi_{0}(x)=\max _{y \in \Phi(x)} \varphi(y)$ for every $x \in D$, the continuity of $\varphi$ and $\Phi$ implies that the marginal function $\varphi_{0}$ is continuous on $D$ by Berge's maximum theorem (see [4, p. 116]).

Let $x, x^{\prime} \in D$ with $x \ll x^{\prime}$ be arbitrary and $\varphi_{0}(x)=\varphi(\bar{x})$ for some $\bar{x} \in C \backslash\{c\}$. Since $x \ll x^{\prime}$, there exists $y \in C$ such that $\bar{x} \ll y \leq x^{\prime}$. By the strict monotonicity of $\varphi$, we have $\varphi(\bar{x})<\varphi(y)$. Therefore, $\varphi_{0}(x)<\varphi_{0}\left(x^{\prime}\right)$, that is, $\varphi_{0}$ is strictly monotonic. Let $x, x^{\prime} \in D$ and $t \in[0,1]$ be such that $t x+(1-t) x^{\prime} \in D$ and let $\varphi_{0}(x)=\varphi(\bar{x})$ and $\varphi_{0}\left(x^{\prime}\right)=\varphi\left(\bar{x}^{\prime}\right)$ for some $\bar{x}, \bar{x}^{\prime} \in C$. By the quasiconcavity of $\varphi$, we have $\min \left\{\varphi(\bar{x}), \varphi\left(\bar{x}^{\prime}\right)\right\} \leq \varphi\left(t \bar{x}+(1-t) \bar{x}^{\prime}\right)$. Because $t \bar{x}+(1-t) \bar{x}^{\prime} \leq t x+(1-t) x^{\prime}$, we have, by the definition of $\varphi_{0}$, that $\varphi\left(t \bar{x}+(1-t) \bar{x}^{\prime}\right) \leq \varphi_{0}\left(t x+(1-t) x^{\prime}\right)$. The last two inequalities imply that $\min \left\{\varphi_{0}(x), \varphi_{0}\left(x^{\prime}\right)\right\}=\min \left\{\varphi(\bar{x}), \varphi\left(\bar{x}^{\prime}\right)\right\} \leq \varphi_{0}\left(t x+(1-t) x^{\prime}\right)$. Thus, $\varphi_{0}$ is quasiconcave on $D .^{3}$

\footnotetext{
${ }^{3}$ Here, $D$ is not a convex set, but the definition of quasiconcavity can be generalized on nonconvex sets as follows: $\varphi: D \rightarrow \mathbb{R}$ is quasiconcave if for every $x, x^{\prime} \in D$ and $t \in[0,1]$ such that $t x+(1-t) x^{\prime} \in D$, we have $\min \left\{\varphi(x), \varphi\left(x^{\prime}\right)\right\} \leq \varphi\left(t x+(1-t) x^{\prime}\right)$.
} 
We now extend function $\varphi_{0}$ to $\mathbb{R}_{+}^{m}$ by setting:

$$
\hat{\varphi}(x):= \begin{cases}\varphi_{0}(x) & \text { if } x \in D, \\ \prod_{k=1}^{m}\left(x_{k}-c_{k}\right)+\varphi_{0}(c) & \text { otherwise }\end{cases}
$$

It is easy to verify that $\hat{\varphi}$ preserves the continuity and strict monotonicity properties of $\varphi$. To demonstrate the quasiconcavity of $\hat{\varphi}$, let, for instance, $x \in D$ and $x^{\prime} \in c+\mathbb{R}_{++}^{m}$, and assume that $y=t x+(1-t) x^{\prime} \in D$ for $t \in[0,1]$. We then have $\hat{\varphi}(x) \leq \hat{\varphi}(x \vee y)=\hat{\varphi}(y) \leq \hat{\varphi}(c) \leq \hat{\varphi}\left(x^{\prime}\right)$, where $x \vee y$ is the coordinatewise maximum of $x$ and $y$ in $\mathbb{R}^{m}$. Hence, $\min \left\{\hat{\varphi}(x), \hat{\varphi}\left(x^{\prime}\right)\right\}=\hat{\varphi}(x) \leq \hat{\varphi}(y)$. The other cases can be checked easily.

Since we have assumed that the component measures of $\vec{\mu}=\left(\mu_{1}, \ldots, \mu_{m}\right)$ are mutually absolutely continuous, for every $x \in \mathcal{R}(\vec{\mu})$ with $x \neq \vec{\mu}(\Omega)$, we have $x \ll \vec{\mu}(\Omega)$. Taking into account this observation, Lemma 5.1 can be deduced from Proposition A.1.

If $\varphi: C \rightarrow \mathbb{R}$ is continuous, concave and monotonic, then an extension $\tilde{\varphi}: \mathbb{R}_{+}^{m} \rightarrow \mathbb{R}$ of $\varphi$, preserving its properties, is given by

$$
\tilde{\varphi}(x)=\max \{\varphi(y) \mid y \in C, y \leq x\} .
$$

This extension was suggested by Einy et al. [11]. Unfortunately, when $\varphi$ is continuous, quasiconcave and strictly monotonic, the extension $\tilde{\varphi}$ to $\mathbb{R}_{+}^{m}$ does not necessarily preserve its properties (strict monotonicity might be violated). We have exploited this extension not on the entire domain $\mathbb{R}_{+}^{m}$, but on $D$ in the proof of Proposition A.1. For a general treatment of extensions of continuous, (strictly) monotonic functions, see Hüsseinov [16].

\section{References}

[1] E. Akin, Vilfred Pareto cuts the cake, J. Math. Econom. 24 (1995) 23-44.

[2] J.-P. Aubin, Cooperative fuzzy games, Math. Oper. Res. 6 (1981) 1-13.

[3] J.-P. Aubin, Mathematical Methods of Game and Economic Theory, Revised Edition, North-Holland, Amsterdam, 1982.

[4] C. Berge, Topological Spaces: Including a Treatment of Multi-Valued Functions, Vector Spaces and Convexity, Oliver \& Boyd, Edinburgh, 1963.

[5] T.F. Bewley, Existence of equilibria in economies with infinitely many commodities, J. Econom. Theory 4 (1972) 514-540.

[6] T.F. Bewley, The equality of the core and the set of equilibria in economies with infinitely many commodities and a continuum of agents, Int. Econom. Rev. 14 (1973) 383-394.

[7] M. Dall'Aglio, The Dubins-Spanier optimization problems in fair division theory, J. Comput. Appl. Math. 130 (2001) 17-40.

[8] N. Dunford, J.T. Schwartz, Linear Operators, Part I: General Theory, John Wiley \& Sons, New York, 1958.

[9] L.E. Dubins, E.H. Spanier, How to cut a cake fairly, Am. Math. Monthly 68 (1961) 1-17.

[10] A. Dvoretsky, A. Wald, J. Wolfowitz, Relations among certain ranges of vector measures, Pac. J. Math. 1 (1951) 59-74.

[11] E. Einy, D. Moreno, B. Shitovitz, The core of a class of non-atomic games which arise in economic applications, Int. J. Game Theory 28 (1999) $1-14$.

[12] M. Florenzano, Edgeworth equilibria, fuzzy core, and equilibria of a production economy without ordered preferences, J. Math. Anal. Appl. 153 (1990) 18-36.

[13] P.R. Halmos, Measure Theory, Van Nostrand, New York, 1950.

[14] F. Hüsseinov, Interpretation of Aubin's fuzzy coalitions and their extension: relaxation of finite exchange economics, J. Math. Econom. 23 (1994) 499-516.

[15] F. Hüsseinov, Existence of the core in a heterogeneous divisible commodity exchange economy, Int. J. Game Theory 37 (2008) $387-395$.

[16] F. Hüsseinov, Monotonic Extension, Department of Economics, Bilkent University, Mimeo, 2010 /http://www.bilkent.edu.tr/ economics/ papers.htm $\rangle$.

[17] F. Hüsseinov, A theory of a heterogeneous divisible commodity exchange economy, J. Math. Econom. 47 (2011) 54-59.

[18] J. Lindenstrauss, A short proof of Liapounoff's convexity theorem, J. Math. Mech. 15 (1966) 971-972.

[19] A. Lyapunov, Sur les fonctions-vecteurs complètement additives, Bull. Acad. Sci. URSS. Sér. Math. 4 (1940) $465-478$ (in Russian).

[20] A. Mas-Colell, W.R. Zame, Equilibrium theory in infinite dimensional spaces, in: W. Hildenbrand, H. Sonnenschein (Eds.), Handbook of Mathematical Economics, vol. IV, Elsevier, North-Holland, 1991, pp. 1835-1898.

[21] N. Sagara, M. Vlach, Representation of preference relations on $\sigma$-algebras of nonatomic measure spaces: convexity and continuity, Fuzzy Sets Syst. 160 (2009) 624-634.

[22] N. Sagara, M. Vlach, Convex functions on $\sigma$-algebras of nonatomic measure spaces, Pac. J. Optim. 6 (2010) 89-102.

[23] N. Sagara, M. Vlach, Convexity of the lower partition range of a concave vector measure, Adv. Math. Econ. 13 (2010) 155-160.

[24] N. Sagara, M. Vlach, A new class of convex games and the optimal partitioning of measurable spaces, Int. J. Game Theory 40 (2011) 617-630.

[25] H.E. Scarf, The core of an $N$ person game, Econometrica 35 (1967) 50-69.

[26] K. Yosida, E. Hewitt, Finitely additive measures, Trans. Am. Math. Soc. 72 (1952) 46-66. 